\title{
Heavy Rydberg states: large amplitude vibrations $\uparrow$
}

\author{
Adam Kirrander, (DD *a Christian Jungen, ${ }^{b}$ Robert J. Donovan ${ }^{a}$ \\ and Kenneth P. Lawley ${ }^{a}$
}

Received 21st May 2018, Accepted 21st June 2018

DOI: $10.1039 / c 8 f d 00096 d$

Extremely large vibrational amplitude $(\approx 8700$ a.u.) heavy Rydberg levels in the $\mathrm{H}^{1}{ }^{1} \Sigma_{\mathrm{g}}^{+}$state, located only $25 \mathrm{~cm}^{-1}$ below the ion-pair dissociation limit, are reported. The calculations are done using a hybrid log derivative/multichannel quantum defect approach that accounts for predissociation and is capable of dealing with any number of long-range closed channels, and of providing positions and widths for the heavy Rydberg resonances. In this case, resonance positions can be reproduced qualitatively using a simple diabatic model (however, the resonance widths cannot). Absolute quantum defects are derived for the vibrational series ranging from $\nu=0$ to $\nu=2010$. The influence of the Coulomb potential and continuity of heavy Rydberg behavior throughout the ${ }^{1} \Sigma_{\mathrm{g}}^{+}$manifold of states is demonstrated.

\section{Introduction}

The electrostatic Coulomb potential between particles of opposite charge can support extremely long-range states in atoms and molecules. For instance, in a recent experiment, $\mathrm{a} \mathrm{Rb}$ atom within a Bose-Einstein condensate was excited to an electronic $\left(\mathrm{Rb}^{+} \mathrm{e}^{-}\right)$Rydberg state with a mean radius of orbit close to $4 \mu \mathrm{m}$, thus exceeding the short axis of the entire 65000 atom condensate. ${ }^{1}$

The vibrational levels of ionically bound states in diatomic molecules are known to form Rydberg series ${ }^{2,3}$ and such vibronic states are now sometimes referred to as 'heavy Rydberg' states. ${ }^{4-8}$ Heavy Rydberg (HR) states are analogous of electronic Rydberg states but with the Rydberg electron replaced by a negative ion. Thus both the center of mass and the reduced mass of HR states differ significantly from those of electronic Rydberg states. However, in either case a system bound by a Coulomb potential at long range will support an infinite number of bound levels and the vibrational levels of a HR state will form a Rydberg series with well-defined effective quantum numbers $n^{*}$ and quantum

${ }^{a}$ EaStCHEM, School of Chemistry, University of Edinburgh, David Brewster Road, EH9 3FJ Edinburgh, UK. E-mail: Adam.Kirrander@ed.ac.uk; Tel: +44 (0)131 6504716

${ }^{b}$ Department of Physics and Astronomy, University College London, London WC1E 6BT, UK

$\dagger$ Electronic supplementary information (ESI) available. See DOI: 10.1039/c8fd00096d 
defects. The vibrational quantum number $\nu$ is a measure of the number of nodes in a given vibrational state and simply replaces the radial quantum number $n$ normally associated with electronic Rydberg states; ${ }^{3}$ note that $\nu$ is a node count so $\nu+1=n$.

HR states have attracted much recent interest and substantial progress has been made in their identification. The ion-pair (IP) states of molecular hydrogen, the hydrogen halides, diatomic halogens and several other molecules exhibit extended vibrational progressions that have been shown to be HR in character over much of their range. ${ }^{8-25}$ Heavy Rydberg behaviour is diagnostic of the dominance of the Coulomb potential in a progression of vibrational levels. The large electronic transition moments associated with valence $\leftrightarrow$ IP state transitions (which must always be parallel), when combined with good Franck-Condon factors, makes ion-pair states effective doorway states for photochemical reactions involving charge transfer. In homonuclear HR states a large transition dipole connects $\mathrm{g} / \mathrm{u}$ pairs of HR states that correlate with the same ionic products and this can lead to amplified spontaneous emission.

There is an intimate connection between ion-pair states and electronic Rydberg states that lie in the same energy region. Their electronic configurations are related by a one or two-electron transfer between molecular orbitals. Asaro and Dalgarno pointed out in 1985 (ref. 2) that the vibrational states in LiF would acquire Rydberg character with increasing excitation. The year before, Mies pioneered the idea that multichannel quantum defect theory (MQDT, see e.g. ref. 26) could be applied to vibrational states in diatomics, ${ }^{27,28}$ and in 1988 published a paper that explicitly discussed the Rydberg-like properties of rotational-vibrational states in alkalihalides. ${ }^{3}$ Around the same time, Zewail and co-workers published their wave packet experiments on $\mathrm{NaI},{ }^{29,30}$ which were almost immediately followed up by time-dependent calculations..,31, ${ }^{31}$ the experiment, a surprisingly strong dependence on the excitation energy was observed for the lifetime of the wave packet. Chapman and Child eventually demonstrated that in the energy domain, this could be understood in terms of a periodic interferometric modulation of the widths of the states constituting the wave packets. ${ }^{33}$ Later, this modulation, which can span several orders of magnitude, was shown to cause Fano $q$-parameter reversals in lineshapes, ${ }^{34,35}$ and more recently, was proposed as the basis for the excitation of long-range ion-pair states in ultracold Rb gas. ${ }^{36}$

The present article is inspired by the observation of photoexcited HR states with high principal quantum number $(n>1000)$ by Ubachs et al., ${ }^{4,5,7,8}$ predominantly in $\mathrm{H}_{2}$. Molecular hydrogen has attracted intense interest from both theoreticians and spectroscopists over many decades. ${ }^{8,9,11,12,14,15,19-21,24,25}$ The potential energy curves for many of the electronic states of $\mathrm{H}_{2}$ below the ionisation energy can now be calculated to high precision and there is close agreement between experiment and theory for the rovibronic term-values of most of the isotopomers which include mass-dependent non-adiabatic corrections. ${ }^{15}$ Mixing between HR and electronic Rydberg states provides doorway states that give access to the HR manifold. We present close-coupled channel calculations in $\mathrm{H}_{2}$, using a hybrid $\log$ derivative/MQDT method ${ }^{\mathbf{1 9 2 0}}$ in an energy range that corresponds to principal quantum numbers $n \approx 2000$. These HR states are embedded in the ionization and dissociation continua. We account for the non-adiabatic couplings to the neutral states of $\mathrm{H}_{2}$, leading to dissociation into neutral atoms, and focus on the 
high energy long-range states between the $n=3$ and the ion-pair dissociation limit. This is the region employed for threshold ion-pair production spectroscopy (TIPS) and zero ion kinetic energy (ZIKE) techniques, and is of interest to astrophysicists, especially as it closely relates to the process of mutual neutralization, $\mathrm{H}^{+}+\mathrm{H}^{-} \rightarrow \mathrm{H}(n \mathrm{l})+\mathrm{H}\left(n^{\prime} \mathrm{l}^{\prime}\right)$, recently discussed by Larson et al. ${ }^{37}$

\section{Properties of Rydberg states}

HR states are bound by the long-range ion-pair potential, ${ }^{38}$

$$
E^{\mathrm{ion}}(R)=D_{\mathrm{A}^{+} \mathrm{B}^{-}}-\frac{1}{R}-\frac{\alpha_{\mathrm{A}^{+}}+\alpha_{\mathrm{B}^{-}}}{2 R^{4}},
$$

where $R$ is the internuclear distance, $D_{\mathrm{A}^{+} \mathrm{B}^{-}}$is the ion-pair dissociation energy, and $\alpha_{\mathrm{A}^{+}}$and $\alpha_{\mathrm{B}^{-}}$are the polarizabilities of the two ions respectively. Atomic units are used throughout. The dominant term in $E^{\text {ion }}(R)$ is the Coulomb potential, $1 / R$, which supports an infinite number of bound states and whose properties underpin the scaling laws shared by all Rydberg systems. ${ }^{39}$ The energies of bound Rydberg states are given by the Rydberg formula,

$$
E_{\mathrm{n}}=D_{\mathrm{A}^{+} \mathrm{B}^{-}}-\frac{\left[2 h c \mathrm{Ry}_{\infty}\right]\left(M_{\mathrm{AB}} / m_{\mathrm{e}}\right)}{2(n-\mu(E))^{2}},
$$

where $n$ is the principal quantum number and $\mu(E)$ is the quantum defect. The quantity in brackets, $\left[2 h c \mathrm{Ry}_{\infty}\right]$, corresponds to one atomic unit of energy. $\mathrm{Ry}_{\infty}$ is the Rydberg constant for an electron associated with an infinite-mass positive charge, and $\left(M_{\mathrm{AB}} / m_{\mathrm{e}}\right)$ is the mass scaling factor given by the ratio of the reduced mass $M_{\mathrm{AB}}$ of $\mathrm{A}^{+} \mathrm{B}^{-}$and the electron mass $m_{\mathrm{e}}$. The quantum defect, $\mu(E)$, gives the shift in the position of each Rydberg energy level compared to the pure Coulomb potential (see discussion below).

All scaling laws for Rydberg states originate from the properties of the Coulomb potential and the Rydberg formula. Since the energy levels scale as $n^{-2}$, the level spacing scales as $n^{-3}$ (with the same scaling implied for line widths and conversely $n^{3}$ for lifetimes). The size of the Rydberg states scales as the classical outer turning point $R_{\mathrm{tp}}$,

$$
R_{\mathrm{tp}} \approx \frac{2 n^{2}}{\left(M_{\mathrm{AB}} / m_{\mathrm{e}}\right)},
$$

with the same $n^{2}$ scaling of the dipole moment.

It is important to note the effect of the reduced mass $M_{\mathrm{AB}}$ in eqn (2) and (3), which is illustrated in Fig. 1. As a consequence of this mass scaling, heavy Rydberg states which by definition have $\left(M_{\mathrm{AB}} / m_{\mathrm{e}}\right) \gg 1$ are more compact and have a larger proportion of the wave function contained in the short-range interaction region for a given $n$. The mass scaling also has an obvious influence on the distribution of energy levels, as illustrated for the $\mathrm{H}$-atom and $\mathrm{H}^{+} \mathrm{H}^{-}$in Fig. 1. In electronic Rydberg states, represented here by the $\mathrm{H}$-atom, almost all energy levels occupy a narrow band just below the ionization limit, while the HR states in $\mathrm{H}^{+} \mathrm{H}^{-}$ occupy a much wider energy band.

The quantum defect $\mu(E)$ in eqn (2) can be related to the asymptotic phase shift in the scattering of the two opposite-charge fragments, ${ }^{26}$ with non-zero quantum defects $\mu(E) \neq 0$ indicative of shorter range interactions. Consider for instance 

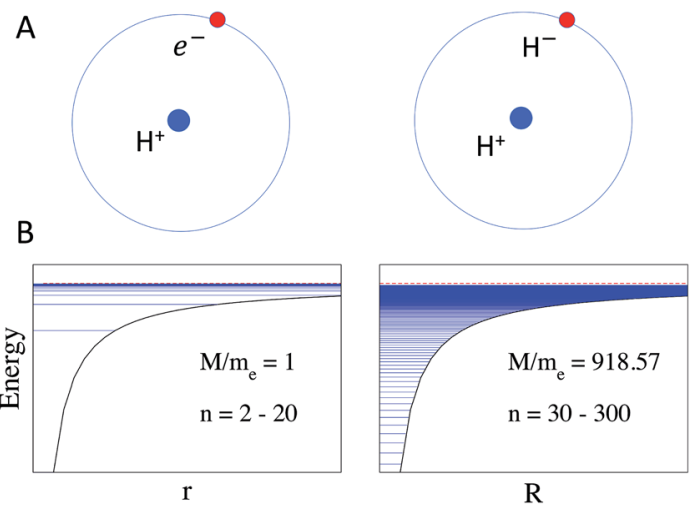

Fig. 1 (A) Schematic of Rydberg states in the $\mathrm{H}$-atom (left) and in the $\mathrm{H}^{+} \mathrm{H}^{-}$heavy Rydberg system (right) in which $\mathrm{H}^{-}$is assumed to be a point charge. (B) The effect of the increased mass on Rydberg energy levels is demonstrated by the distribution of states according to the Rydberg formula using the effective mass of the $\mathrm{H}$-atom on the left with $M_{\mathrm{AB}}=m_{\mathrm{e}}=1$ and $n=2-20$, and the $\mathrm{H}_{2}$ molecule on the right with $M_{A B}=918$ and $n=30-300$. Note that for the $\mathrm{H}_{2}$ molecule the states would be perturbed by short-range repulsive interactions, not included in this schematic (see later). Energy levels are indicated by horizontal lines.

the first electronically excited state of molecular hydrogen, the $\mathrm{H}_{2}\left(\mathrm{~B}^{1} \Sigma_{\mathrm{u}}^{+}\right)$state, which has an ionic configuration $\mathrm{H}^{+} \mathrm{H}^{-}$derived from the molecular orbital configuration $\sigma_{\mathrm{g}}^{1} \sigma_{\mathrm{u}}^{1}$ for radial separations $R<10$ a.u. where it crosses the atom-pair asymptote $\mathrm{H}(1 \mathrm{~s})+\mathrm{H}(2 \mathrm{~s})$ leading to dissociation. The potential below this crossing is thus coulombic albeit modified by a small polarization term, until, on the inner branch of the potential, nuclear repulsion and electron-pair repulsion begin to dominate at short range. The resulting inner wall, present in all molecular ionpair potentials, restricts the available phase space for vibration and results in large negative quantum defects. ${ }^{3}$ This is in contrast to the positive quantum defects for electronic Rydberg series in which the Rydberg electron can penetrate through inner valence shells to experience an increased effective nuclear charge giving a positive quantum defect. The energy dependence of the quantum defect generally decreases with increasing principal quantum number, as the Coulomb waves become increasingly similar in the interaction region, although the onset of this effect is slower in HR states due to mass scaling. ${ }^{19}$ As a general remark in the context of $\mathrm{H}_{2}$, note that HR states can only appear in the singlet manifolds of states, since the singlet $\mathrm{H}^{-}\left(1 \mathrm{~s}^{2}\right)$ negative ion is the only stable form of the anion.

\section{Theory}

\subsection{Overview}

The HR states can be regarded as a long-range collision complex between two opposite-charge fragments. The complex can decay by predissociation, radiative decay, or ionisation. The following approach considers predissociation, with radiative decay slow enough to be ignored in the first approximation and the coupling to ionisation having a comparatively small influence on line positions, as will be argued later. The problem is treated using a hybrid log derivate-MQDT approach applied across the three distinct regions shown in Fig. 2. The inner 


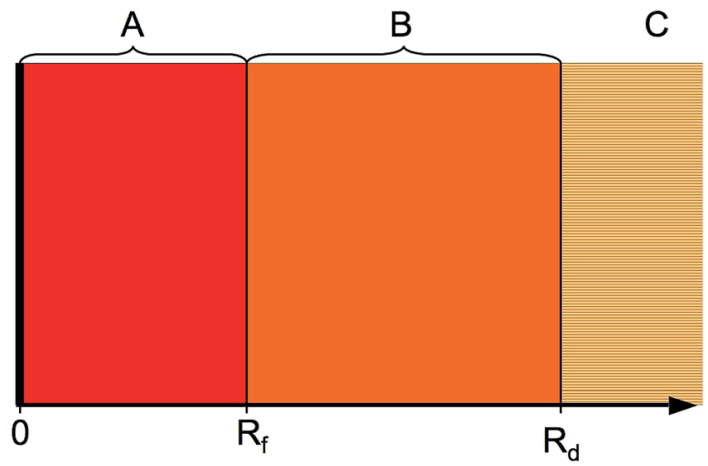

Fig. 2 The calculations proceed through three regions. The inner interaction region $\mathrm{A}(0<$ $R<R_{\mathrm{f}}$ ) has strong non-adiabatic couplings and we solve the close-coupled equations using the log derivative method. The intermediate region $\mathrm{B}\left(R_{\mathrm{f}}<R<R_{\mathrm{d}}\right)$ has no interchannel couplings, but some channels contain long-range potentials capable of supporting large-amplitude resonances. These asymptotically closed channels are eliminated by an MQDT procedure. Finally, region $C\left(R>R_{\mathrm{d}}\right)$ is the asymptotic region.

region $\mathrm{A}$ is the interaction region, where the nonadiabatic interaction between the colliding fragments must be accounted for (using numerical integration of the close-coupled equations by the log derivative method, Section 3.2), the intermediate region B is the long-range region which supports heavy Rydberg resonances in the closed ion-pair channel (treated using an MQDT approach for channel elimination, Section 3.3). Finally, in the asymptotic region C, all interactions between the collision fragments have ceased.

\subsection{Interaction region}

We consider first the interaction region (region A: $0<R<R_{\mathrm{f}}$ in Fig. 2) corresponding to the collision complex. The theory of nonadiabatic atom-atom collisions is well-established (see e.g. ref. 37, 40 and 41). The dynamics are governed by a set of close-coupled partial differential equations,

$$
\left(-\frac{1}{2 M_{\mathrm{AB}}}\left[1 \frac{\mathrm{d}^{2}}{\mathrm{~d} R^{2}}-1 \frac{J(J+1)}{R^{2}}+\boldsymbol{A}(R)+\boldsymbol{B}(R) \frac{\mathrm{d}}{\mathrm{d} R}\right]+\boldsymbol{U}(R)-1 E\right) \tilde{\Psi}(R)=0,
$$

where $M_{\mathrm{AB}}$ is the reduced mass and $\boldsymbol{U}(R)$ contains the $N^{\prime}$ clamped-nuclei electronic energy curves, $E$ is the total energy, and $\boldsymbol{A}(R)$ and $\boldsymbol{B}(R)$ are the $N^{\prime} \times N^{\prime}$ nonadiabatic coupling matrices. The leading first-derivative coupling terms can be eliminated by a Cayley transform,

$$
\left[1 \frac{\mathrm{d}}{\mathrm{d} R}+\boldsymbol{B}(R)\right] \boldsymbol{C}(R)=0,
$$

with boundary condition $\boldsymbol{C}(R \rightarrow \infty)=1$. The matrix $\boldsymbol{C}(R)$ defines the adiabaticdiabatic transformation. This allows us to transform eqn (4) into the standard form,

$$
\boldsymbol{\Psi}^{\prime \prime}(R)=\boldsymbol{W}(R) \boldsymbol{\Psi}(R),
$$

where $\Psi$ is a $N^{\prime} \times N^{\prime}$ matrix, with each column being a linearly independent solution, $\Psi^{\prime \prime}$ indicates the second derivative with respect to $R$, and the matrix $\boldsymbol{W}$ consists of 


$$
\boldsymbol{W}(R)=\frac{2 M_{\mathrm{AB}}}{\hbar^{2}} \boldsymbol{V}(R)-\boldsymbol{k}^{2},
$$

where the $N^{\prime} \times N^{\prime}$ matrix $\boldsymbol{V}$ contains the diagonal potentials (including the angular momentum components) and the off-diagonal coupling elements. The diagonal matrix $\boldsymbol{k}$ contains the asymptotic channel wave vectors $\boldsymbol{k}^{2}=\left(2 M_{\mathrm{AB}} / \hbar^{2}\right) \boldsymbol{\varepsilon}$, where $\varepsilon$ has diagonal elements $\varepsilon_{i}=E-E_{i}$, with $E$ being the total energy and $E_{i}$ being the threshold energy in each channel $i$.

Eqn (6) is solved using the log derivative method, ${ }^{42-44}$ which propagates the log derivative matrix $\boldsymbol{Y}$, defined as

$$
\boldsymbol{Y}(R)=\boldsymbol{\Psi}^{\prime}(R) \boldsymbol{\Psi}^{-1}(R),
$$

where $\Psi^{\prime}$ indicates the first derivative with respect to $R$. Propagation of $\boldsymbol{Y}$ eliminates stability problems that can arise when the integration is started deep inside a classically forbidden region, or if it enters regions where a subset of channels are classically forbidden. Differentiation of eqn (8) and removal of the second derivative using eqn (6) gives a non-linear first order differential equation,

$$
\boldsymbol{Y}^{\prime}(R)=\boldsymbol{W}(R)-\boldsymbol{Y}^{2}(R)
$$

known as the matrix Riccati equation. Since $\boldsymbol{Y}$ is undefined when $\operatorname{det}|\boldsymbol{\Psi}|=0$, standard integration techniques are not applicable and a form of invariant embedding must be used instead (see the extensive literature on the log derivative method, e.g. ref. 42-44).

The matrix is propagated out to the matching radius $R_{\mathrm{f}}$, at which point we assume that the channel interactions have vanished. At that point, the matrix is used to calculate the wave function in the form

$$
\boldsymbol{\Psi}=\boldsymbol{F}-\boldsymbol{G K}
$$

where the wave function $\boldsymbol{\Psi}$ is a $N \times N$ matrix with each column corresponding to one solution, and $\boldsymbol{F}$ and $\boldsymbol{G}$ are diagonal $N \times N$ matrices containing energynormalized Milne functions. ${ }^{45}$ These coincide with the analytic Coulomb and Riccati-Bessel functions once the polarization term in eqn (1) vanishes. The crucial entity is the $N \times N$ reaction matrix $K$, which summarizes all interactions for $R<R_{\mathrm{f}}$, and which is independent of $R$, i.e. $\mathrm{d} K / \mathrm{d} R=0$, in the absence of channel interactions. Since channels closed already at $R_{\mathrm{f}}$ are excluded from $\boldsymbol{\Psi}$, note that $N \leq N^{\prime}$. The matrix $\boldsymbol{K}$ is obtained by

$$
\boldsymbol{K}=\boldsymbol{G}^{-1} \boldsymbol{F}+\boldsymbol{G}^{-1} \boldsymbol{B}^{-1} \boldsymbol{G}^{-1} \boldsymbol{\Omega},
$$

where $\boldsymbol{B}=\boldsymbol{Y}-\boldsymbol{G}^{\prime} \boldsymbol{G}^{-1}$, and only the $N \times N$ (open-open) parts of the matrices are considered in eqn (11) (and eqn (10)), since the components associated with the $N^{\prime}-N$ closed channels have vanished. For energy-normalized Milne wave functions in natural units, the Wronskian matrix is given by $\boldsymbol{\Omega}_{i j}(R)=\pi^{-1} \delta_{i j}$. The calculations of the matrix $\boldsymbol{K}$ (or equivalently $\boldsymbol{S}$ ) would normally constitute the endpoint of the calculation. However, in the presence of HR resonances, the longrange ion-pair potential supports resonances in the external region $\mathrm{B}\left(R_{\mathrm{f}}<R<\right.$ $R_{\mathrm{d}}$ ), the treatment of which will be discussed in the next section. 


\subsection{Intermediate region}

We consider here the situation where the intermediate region (region B: $R_{\mathrm{f}}<R<$ $R_{\mathrm{d}}$ in Fig. 2) contains long-range potentials capable of supporting additional vibrational resonances. Assume that among the $N$ channels in the $R>R_{\mathrm{f}}$ wave function in eqn (10), there exists a subset of $N_{\mathrm{c}}$ asymptotically closed channels, for instance due to the ion-pair Coulomb potential, such that $N=N_{\mathrm{o}}+N_{\mathrm{c}}$, where $N_{\mathrm{o}}$ are the asymptotic channels that remain open for $R>R_{\mathrm{d}}$. The $N_{\mathrm{c}}$ closed channels can be eliminated using multichannel quantum defect theory (MQDT). ${ }^{26,46}$ For energies close to the threshold, where the wave functions in the Coulomb channels extend to large distances, this procedure is more efficient than integrating all $N$ channels to asymptotic distances.

In essence, the elimination procedure uses the $N$ linearly independent solutions given by the column vectors of $\boldsymbol{\Psi}$ in eqn (10) to form $N_{\mathrm{o}}$ superpositions with correct asymptotic boundary conditions. This can be expressed as

$$
\boldsymbol{\Phi}=\Psi Z,
$$

where $\boldsymbol{\Phi}$ is a $N \times N_{\mathrm{o}}$ matrix, $\boldsymbol{\Psi}$ a $N \times N$ matrix, and the coefficient matrix $\boldsymbol{Z}$ is $N \times$ $N_{\mathrm{o}}$. As before, each column is one solution.

Rewriting the matrix $\boldsymbol{K}$ as

$$
\boldsymbol{K}=\mathscr{S} \mathscr{C}^{-1}
$$

where $\mathscr{P}$ and $\mathscr{C}$ are $N \times N$ matrices, and the coefficient matrix $\boldsymbol{Z}$ as

$$
\boldsymbol{Z}=\mathscr{C} \boldsymbol{B},
$$

allows us to express eqn (12) as

$$
\boldsymbol{\Phi}=(\boldsymbol{F}-\boldsymbol{G K}) \boldsymbol{Z}=(\boldsymbol{F} \boldsymbol{C}-\boldsymbol{G} \mathscr{P}) \boldsymbol{B} .
$$

The component of the wave function given by eqn (15) for channel $i$ and solution $j$ is

$$
\boldsymbol{\Phi}_{i j}=\pi^{-1 / 2} \alpha_{i}\left(\varepsilon_{i}, R\right) \sum_{i^{\prime}}^{N}\left(\sin \boldsymbol{\Psi}_{i}\left(\varepsilon_{i}, R\right) \mathscr{C}_{i i^{\prime}}+\cos \boldsymbol{\Phi}_{i}\left(\varepsilon_{i}, R\right) \mathscr{T}_{i i^{\prime}}\right) B_{i^{\prime} j},
$$

where $\phi_{i}\left(\varepsilon_{i}, R \rightarrow \infty\right)=\beta_{i}\left(\varepsilon_{i}\right)$ for closed channels, with $\beta_{i}\left(\varepsilon_{i}\right)$ the accumulated phase $R: R_{\mathrm{f}} \rightarrow \infty$ at energy $\varepsilon_{i} \cdot \downarrow$ At large distances $R \rightarrow \infty$ the wave function must be zero in closed channels, meaning that

$$
\sum_{i^{\prime}}^{N}\left(\sin \beta_{i} \mathscr{C}_{i i^{\prime}}+\cos \beta_{i} \mathscr{S}_{i i^{\prime}}\right) B_{i^{\prime} j}=0
$$

which in matrix form is

$$
(\sin \beta \mathscr{C}+\cos \beta \mathscr{P}) \boldsymbol{B}=0,
$$

with $\sin \beta$ and $\cos \beta$ diagonal $N \times N$ matrices with elements $(\sin \beta)_{i i}=\sin \beta\left(\varepsilon_{i}\right)$.

For asymptotically open channels, eqn (16) has $\phi_{i}\left(\varepsilon_{i}, R \rightarrow \infty\right)=k_{i} R+\delta_{i}$, assuming the asymptotic potential is constant. We can rewrite the sum as 


$$
\sin \phi_{i} \underbrace{\left(\sum_{i^{\prime}}^{N} \mathscr{C}_{i i^{\prime}} B_{i^{\prime} j}\right)}_{T_{i} \cos \pi \tau}+\cos \phi_{i} \underbrace{\left(\sum_{i^{\prime}}^{N} \mathscr{S}_{i i^{\prime}} B_{i^{\prime} j}\right)}_{T_{i} \sin \pi \tau}=T_{i} \sin \left(\phi_{i}+\pi \tau\right) .
$$

To obtain a condition similar in form to the closed channel condition in eqn (17), while ensuring that each open channel has the same asymptotic phase shift $\pi \tau$, we can define

$$
\begin{aligned}
0 & =-T_{i} \cos \pi \tau \sin \pi \tau+T_{i} \sin \pi \tau \cos \pi \tau \\
& =\sum_{i^{\prime}}^{N}\left[\mathscr{C}_{i i^{\prime}} \sin (-\pi \tau)+\mathscr{S}_{i i^{\prime}} \cos (-\pi \tau)\right] B_{i^{\prime} j},
\end{aligned}
$$

which in matrix form becomes

$$
[\sin (-\pi \tau) \mathscr{C}+\cos (-\pi \tau) \mathscr{P}] \boldsymbol{B}=0
$$

The unknowns in eqn (18) and (21) are the $N \times N_{\mathrm{o}}$ coefficient matrix $\boldsymbol{B}$ and the $N_{\mathrm{o}}$ eigenphases $\tau$. A numerically convenient method to obtain all these in a single calculation is to combine eqn (18) and (21) in the form of a generalized eigenvalue equation,

$$
\boldsymbol{\Gamma} \boldsymbol{B}=\tan \pi \tau \boldsymbol{\Lambda} \boldsymbol{B},
$$

where the $N \times N$ matrices $\boldsymbol{\Gamma}$ and $\boldsymbol{\Lambda}$ are defined as

$$
\begin{array}{lll}
\boldsymbol{\Gamma}_{i i^{\prime}}=\sin \beta_{i} \mathscr{C}_{i i^{\prime}}+\cos \beta_{i} \mathscr{S}_{i i^{\prime}}, & & i \in \text { closed } \\
\boldsymbol{\Gamma}_{i i^{\prime}}=\mathscr{S}_{i i^{\prime}}, & & i \in \text { open } \\
\boldsymbol{\Lambda}_{i i^{\prime}}=0, & & i \in \text { closed } \\
\boldsymbol{\Lambda}_{i i^{\prime}}=\mathscr{C}_{i i^{\prime}}, & & i \in \text { open } .
\end{array}
$$

The main remaining steps are to normalize the continuum eigenvectors in $\boldsymbol{B}$ and to calculate the scattering matrix $\boldsymbol{S}$. According to the definitions of $T$ in eqn (19), we have that

$$
\begin{aligned}
T_{i j} & =T_{i j} \cos ^{2}\left(\pi \tau_{\rho}\right)+T_{i j} \sin ^{2}\left(\pi \tau_{\rho}\right) \\
& =\sum_{i^{\prime}}^{N}\left[\cos \left(\pi \tau_{\rho}\right) \mathscr{C}_{i i^{\prime}}+\sin \left(\pi \tau_{\rho}\right) \mathscr{S}_{i i^{\prime}}\right] B_{i^{\prime} j}
\end{aligned}
$$

or in matrix form,

$$
\boldsymbol{T}=\left[\cos \left(\pi \tau_{\rho}\right) \mathscr{C}+\sin \left(\pi \tau_{\rho}\right) \mathscr{P}\right] \boldsymbol{B}
$$

The matrix $\boldsymbol{T}$ can be shown to be orthogonal ${ }^{46}$ and becomes unitary, $\boldsymbol{T} \boldsymbol{T}^{\dagger}=1$, if it is normalised according to $\sum_{i}^{N_{0}} T_{i j}^{2}=1, j \in N_{\mathrm{o}}$. This condition fixes the overall normalization of the $N$ coefficients $B_{i j}$ for each $j$.

We can now obtain the $N_{\mathrm{o}} \times N_{\mathrm{o}}$ scattering matrix $\boldsymbol{S}^{-}$for incoming wave boundary conditions, appropriate for photodissociation. Combining the final 
wave function in eqn (15) with the definition of the coefficients $T$ in eqn (19) gives the wave function for solution $j$ in channel $i\left(i, j \in N_{\mathrm{o}}\right)$ as follows,

$$
\begin{aligned}
\Phi_{i j} & =\pi^{-1 / 2} \alpha_{i}(R \rightarrow \infty) T_{i j} \sin \left(\phi_{i}+\pi \tau_{j}\right) \\
& =\frac{1}{2 l} \pi^{-1 / 2} \alpha_{i} T_{i j}\left(\mathrm{e}^{\imath \phi_{\mathrm{i}}+l \pi \tau_{\mathrm{j}}}-\mathrm{e}^{-l \phi_{\mathrm{i}}-l \pi \tau_{\mathrm{j}}}\right) .
\end{aligned}
$$

Henceforth we will use the asymptotic value of the open channel phase, $\phi_{i}\left(\varepsilon_{i}, R \rightarrow \infty\right)=k_{i} R+\delta_{i}$.

The following superposition corresponds to an asymptotic wave function incoming boundary condition,

$$
\begin{aligned}
\Phi_{i j}^{-} & =\sum_{\rho=1}^{N_{\mathrm{o}}} \mathrm{e}^{-l \pi \tau_{\rho}} \Phi_{i \rho} T_{\rho j}{ }^{-1} \mathrm{e}^{-l \delta_{i}} \\
& =\frac{1}{2 l} \pi^{-1 / 2} \alpha_{i}\left[\mathrm{e}^{\imath k_{i} R} \delta_{i j}-\mathrm{e}^{-l k_{i} R}\left(\mathrm{e}^{-l \delta_{i}} \sum_{\rho=1}^{N_{\mathrm{o}}} T_{i \rho} \mathrm{e}^{-l 2 \pi \tau_{\rho}} T_{\rho j}{ }^{-1} \mathrm{e}^{-l \delta_{i}}\right)\right]
\end{aligned}
$$

and we can identify the asymptotic open-open $\left(N_{\mathrm{o}} \times N_{\mathrm{o}}\right)$ scattering matrix $\boldsymbol{S}^{-}(\boldsymbol{E})$ as

$$
\boldsymbol{S}_{i j}{ }^{-}=\mathrm{e}^{-l \delta_{i}}\left(\sum_{\rho=1}^{N_{\mathrm{o}}} T_{i \rho} \mathrm{e}^{-l 2 \pi \tau_{\rho}} T_{\rho j}{ }^{-1}\right) \mathrm{e}^{-l \delta_{j}},
$$

where $\tau_{\rho}$ is the asymptotic phase in channel $i$ and the unitary matrix $\mathbf{T}$ is defined as $\mathbf{T}=\mathbf{C B} \cos \pi \tau+\mathbf{S B} \sin \pi \tau$. The matrix $\boldsymbol{S}^{-}(\boldsymbol{E})$ will have strong energydependence reflective of the sharp long-range resonances, characterized by rapid change in the total eigenphase of the scattering matrix. The positions of the resonances can be obtained from the derivative of the cumulative eigenphase, $\mathrm{d} \sum_{\rho} \pi \tau_{\rho}(E) / \mathrm{d} E$, and the width is obtained from the full width at half maximum (FWHM) for each peak. The positions of the peaks allow us to determine the value of the quantum defect by reference to eqn (2). The shifts compared to the pure Coulomb potential are expressed in terms of either the quantum defect itself, $\mu(E)$, or equivalently the effective quantum number, $n^{*}=n-\mu(E)$.

\section{Calculations}

The $a b$ initio potential energy curves and the non-adiabatic couplings are taken from Wolniewicz et al. ${ }^{12,38}$ for states $2-6^{1} \Sigma_{\mathrm{g}}^{+}$and from Detmer et al. ${ }^{47}$ for $7-$ $9^{1} \Sigma_{\mathrm{g}}^{+}$. The non-adiabatic couplings are particularly strong for $R<20$ a.u. The potential energy curves are shown in Fig. 3. The figure includes the ion-pair potential and the ionisation limit in terms of the $\mathrm{H}_{2}^{+}$ground state. Weakly avoided crossings between the ion-pair potential and the adiabatic potential energy curves appear at $R \approx 36$ a.u. $(\mathrm{H}(1 \mathrm{~s})+\mathrm{H}(3 \mathrm{l})$ dissociation limit) and $R \approx 280$ a.u. $(\mathrm{H}(1 \mathrm{~s})+\mathrm{H}(4 \mathrm{l})$ dissociation limit). Table 1 includes indicative principal quantum numbers to provide a sense of the outer turning point on the ion-pair potential for the vibrational states at corresponding energies.

At large internuclear distances $(R>10$ a.u.) the ion-pair (HR) states are accurately described by the Coulomb interaction energy between the positive and 


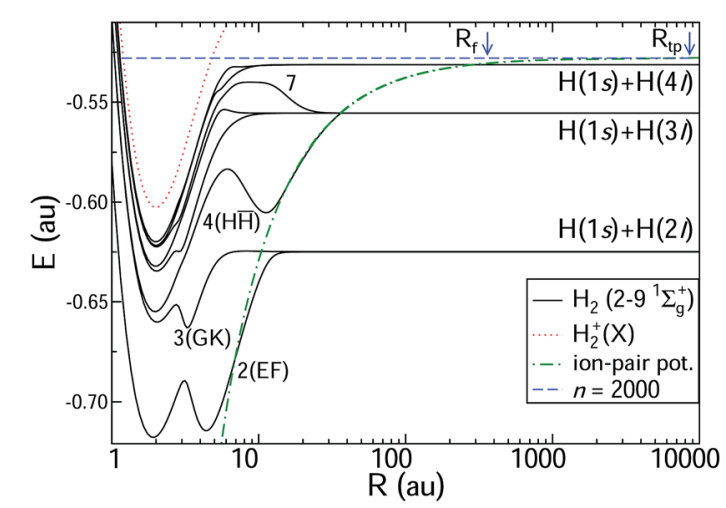

Fig. 3 Potential energy curves for $\mathrm{H}_{2}$ of ${ }^{1} \Sigma_{\mathrm{g}}^{+}$symmetry. The potential energy curves are taken from Wolniewicz et al. for states 2-6 (ref. 12 and 38) and from Detmer et al. for states 7-9.47 The $\mathrm{H}_{2}^{+}$ground-state potential is also included, as is the ion-pair potential. The position of the $n=2000 \mathrm{HR}$ state is indicated by a vertical line, with the classical turning point $R_{\mathrm{tp}}$ at 8700 a.u. and the position of $R_{\mathrm{f}}$ (see Fig. 2). The unusual barrier shape in the region of $R \approx 10$ on the excited potential energy curves can be understood using a simple Fermi model as contact potential interactions between a colliding $\mathrm{H}(1 \mathrm{~s})$ atom and an almost-Rydberg $\mathrm{H}(3 \mathrm{l})$ atom. ${ }^{48,49}$ Note that the scale of the axis for the internuclear distance $R$ is logarithmic. For $R>60$ a.u. the potential energy curves are continued analytically.

Table 1 Table of features in ${ }^{1} \Sigma_{g}^{+}$potential energy curves. For each feature we include the principal quantum number $n^{*}$ and the classical outer turning point in the ion-pair potential at the corresponding energy

\begin{tabular}{lll}
\hline Feature & $n^{*}$ & $R_{\mathrm{tp}}$ (a.u.) \\
\hline $\mathrm{H}(1 \mathrm{~s})+\mathrm{H}(21)$ dissociation limit & 68.72 & 11.08 \\
$\mathrm{H}(1 \mathrm{~s})+\mathrm{H}(31)$ dissociation limit & 128.52 & 36.05 \\
$\mathrm{H}(1 \mathrm{~s})+\mathrm{H}(41)$ dissociation limit & 362.30 & 286 \\
Outer well state 7 (at $R=33.8$ a.u.) & 128.68 & 36.13 \\
Barrier on state 7 (at $R=8.7$ a.u.) & 193.53 & 81.56 \\
At $n=100$ energy & 100 & 22 \\
At $n=200$ energy & 200 & 87 \\
At $n=1000$ energy & 1000 & 2177 \\
At $n=2000$ energy & 2000 & 8709
\end{tabular}

negative ion given by eqn (1) with the ion-pair dissociation energy $D_{\mathrm{H}^{+} \mathrm{H}^{-}}=$ -0.527751014 a.u. (ref. 38 and 50) and the polarizability of $\mathrm{H}^{-}\left(1 \mathrm{~s}^{2}\right) \alpha_{\mathrm{H}^{-}}=211.897$ (a.u.) ${ }^{3}$ (see ref. 51 and also footnote§). The reduced mass for $\mathrm{H}_{2}$ is $M_{\mathrm{H}_{2}} \approx 918.0764$ a.u. ${ }^{53}$

For the model diabatic calculation, we construct an effective diabatic potential $E^{\text {ion,diab }}(R)$ from the $\mathrm{HH}^{1} \Sigma_{\mathrm{g}}$ ab initio adiabatic state calculated by Wolniewicz ${ }^{38}$ and the asymptotic ion-pair potential in eqn (1). ${ }^{50,51}$ The potentials are merged as follows,

$\S$ Note that the electron affinity can be calculated as $E_{\mathrm{ea}}=D_{\mathrm{H}^{+} \mathrm{H}^{-}}-E_{\mathrm{H}^{-}}-E_{\mathrm{H}}$ and that the value of the electron affinity obtained this way differs somewhat from the value -0.0277196153 a.u. reported by Radtzig et al. ${ }^{52}$ 


$$
E^{\mathrm{ion}, \mathrm{diab}}(R)=\gamma(R) E^{\mathrm{ion}}(R)+(1-\gamma(R)) E^{\mathrm{HH}}(R),
$$

where the mixing function $\gamma(R)$ is defined as $\gamma(R<33)=0,0<\gamma(33<R<35.5)=$ $(R-33) / 2.5<1$, and finally $\gamma(R>35.5)=1$. Resonance positions on the diabatic model potential are determined using the shooting method, while all other calculations are done using the full machinery described in Sections 3.2 and 3.3.

\section{Results and discussion}

Tables of calculated long-range vibrational HR resonances for $J=0,1$, and 2 in the energy interval $[-0.554933,-0.536094]$ a.u., corresponding to principal quantum numbers $n=130-235$, are provided in the ESI, $\uparrow$ listing the energy, effective quantum number and FWHM width of each resonance. This energy region corresponds to previous measurements by Ubachs et al. ${ }^{7,8}$ The overall agreement for line (resonance) positions is good. In contrast, the calculated widths, which decrease from $26 \mathrm{~cm}^{-1}$ to $2.4 \mathrm{~cm}^{-1}$ across the range $n=130-235$, are systematically narrower than those observed in experiments. The source of this discrepancy must relate to decay channels other than predissociation that are not included in the present calculations, with ionisation most likely since these states are known to exhibit autoionisation. ${ }^{19}$

The main physics underpinning the progression of line positions across the range $n=160-230$ is assessed by comparison to the simple diabatic model constructed from $\mathrm{HH}^{1} \Sigma_{\mathrm{g}}^{+}$and the ion-pair potential (see Section 4). A comparison between the line positions resulting from the full log derivative-MQDT hybrid calculation and the model in this energy region show that the model follows the pattern of line positions closely, with only a comparatively small and almost constant off-set (Fig. 4).

It therefore appears that a large portion of the energy dependence in the quantum defect, $\mu(E)$, can be attributed to the vibrational wavefunction accumulating phase on the diabatic $\mathrm{H} \overline{\mathrm{H}}$ potential. The model diabatic potential and a bound state on that potential are shown in Fig. 5 . Note that states on the model potential are technically bound states, and that the model in its current form

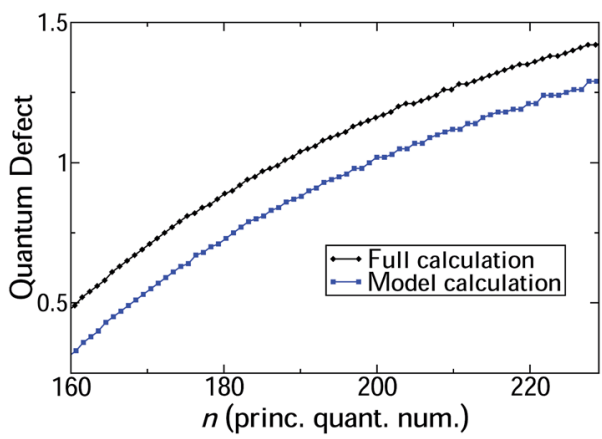

Fig. 4 Quantum defects for $J=0$ HR states in the range $n=160-230$ calculated using the full log derivative/MQDT approach outlined in the article, as well as a simple diabatic model (see text for details). The model calculations follow the trend for the full calculation closely. 


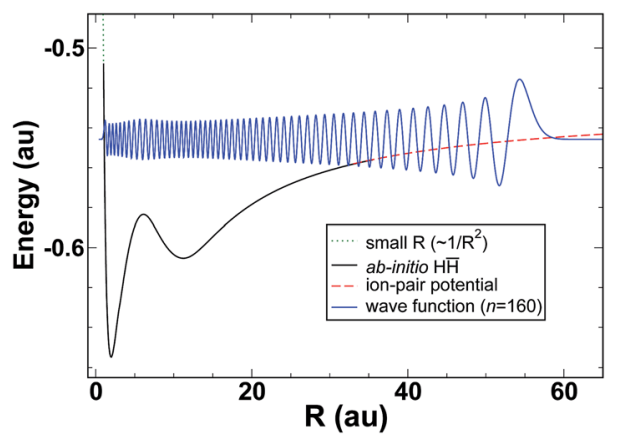

Fig. 5 Effective potential (merged $\mathrm{H} \overline{\mathrm{H}}$ and ion-pair potential) together with the bound $n=$ 160 radial wavefunction.

cannot make predictions of line widths. Given the importance of interferometric lifetime modulation in HR states, and the complexity of the $\mathrm{H}_{2}$ potentials in the interaction region, it is doubtful that a meaningful simplified model could be constructed for the lifetimes (widths).

We now proceed to the part of the HR spectrum just below the ion-pair dissociation limit. Using the hybrid log derivative-MQDT approach we calculate a narrow range with principal quantum number $1990<n<2010$ as shown in Fig. 6 for total angular momenta $J=0,1$, and 2 . The line positions are quite regular, with nearly constant quantum defects $\mu(E)$ across the range. The widths, on the other hand, exhibit modulation across the range, with different variation in line widths for the different angular momenta $J=0,1,2$. The calculated width for the state $n=2000$ is $5 \times 10^{-4} \mathrm{~cm}^{-1}$, corresponding to a lifetime of approximately 10 ns. This is almost an order of magnitude shorter than the observed lifetimes reported by Ubachs et $a l .{ }^{4}$ Since one would normally expect the calculations to over-estimate lifetimes on the basis that not all decay channels are included in the calculations, this lends support to the notion that such high- $n$ HR states have a strong tendency to undergo $J$-mixing by external fields, analogous to the $l$-mixing that occurs in electronic zero electron kinetic energy (ZEKE) spectroscopy.

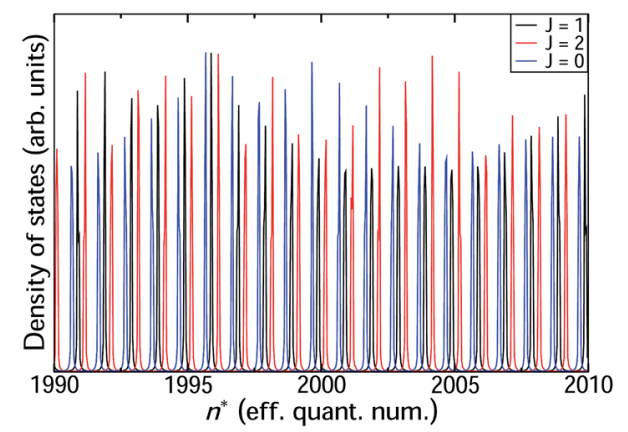

Fig. 6 Progression of heavy Rydberg resonances around the principal quantum number $n=2000$ for $\mathrm{J}=0,1$ and 2 . These states have classical turning points at around $R_{\mathrm{tp}}=8700$ a.u. 
Term values of the vibrational levels supported by the adiabatic $\mathrm{H} \overline{\mathrm{H}}$ potential of Wolniewicz have been calculated up to $\nu=39\left(E_{\mathrm{b}}=8000 \mathrm{~cm}^{-1}\right)$ and we include a complete table of resonances in the energy region $E_{\mathrm{b}}=1800-6000 \mathrm{~cm}^{-1}(\mathrm{ESI} \dagger)$ calculated by solving the close-coupled equations for seven ${ }^{1} \Sigma_{\mathrm{g}}^{+}$states. If the vibrational numbering is known, the absolute quantum defect follows from $-\mu\left(E_{\mathrm{b}}\right)=n^{*}-\nu-1-J$, where $n^{*}=\sqrt{\left(\mathrm{Ry} / E_{\mathrm{b}}\right)}$. Such $\mu\left(E_{\mathrm{b}}\right)$ plots, both from model potentials and experimentally, for a wide range of ion-pair states have been shown to be nearly linear over a wide range of $E_{\mathrm{b}}$ values as the dissociation limit is approached. Thus, if experimental $T_{\mathrm{v}}$ values are used from fragmentary spectra in which the absolute vibrational numbering is not known, $\nu$ is adjusted so that the $\nu\left(E_{\mathrm{b}}\right)$ values for a partial progression lie on the $\mu\left(E_{\mathrm{b}}\right)$ plot extrapolated from regions of known numbering. If the numbering is changed by \pm 1 , clear discontinuities in the sections of the $\mu\left(E_{\mathrm{b}}\right)$ plot result.

The various calculated $\mu\left(E_{\mathrm{b}}\right)$ values for the $\mathrm{H} \overline{\mathrm{H}}$ state are summarized in Fig. 7. The high vibrational levels presented in this paper cover the range $E_{\mathrm{b}}=25.5-$ $25 \mathrm{~cm}^{-1}$ and, if positioned on the extrapolated $\mu\left(E_{\mathrm{b}}\right)$ plot, indicate a quantum defect of -63.8 at $E_{\mathrm{b}} 25 \mathrm{~cm}^{-1}$. The condition $n \gg \mu$ is sometimes taken to define HR behaviour, and this is certainly fulfilled by the levels in Fig. 7. The broader $\mu\left(E_{\mathrm{b}}\right)$ dependence in Fig. 7 indicates that there is no abrupt transition from a HR region to one deeper in the potential well in which the vibrational spacing becomes sensitive to the position of the inner wall of the potential, even after

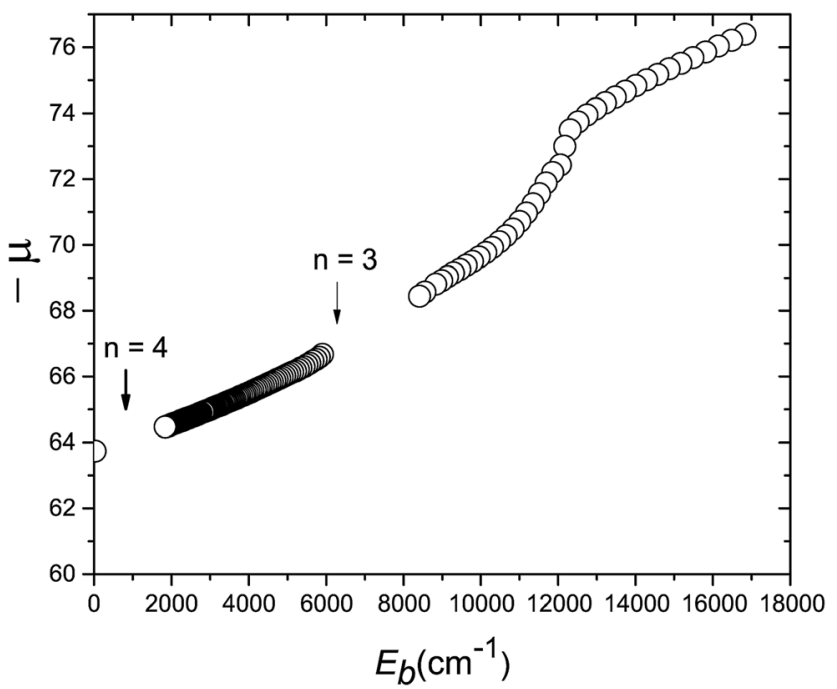

Fig. 7 Quantum defect plot for the vibrational levels of the $\mathrm{H}^{1}{ }^{1} \Sigma_{\mathrm{g}}^{+}$state for $\nu^{\prime}=0$ up to $\nu^{\prime}=2010$. The absolute quantum defects, $-\mu\left(E_{\mathrm{b}}\right)$, are plotted as a function of the binding energy $E_{\mathrm{b}}$ below the ion-pair dissociation limit. Previously calculated term values for $\nu^{\prime}=$ 0-40 are taken from ref. 54. The break in slope at $E_{\mathrm{b}} \approx 13000 \mathrm{~cm}^{-1}$ corresponds to the top of the barrier between the electronic and heavy Rydberg potentials $\left(\nu^{\prime}=16\right)$. Calculated resonances lie between $E_{\mathrm{b}}=2000$ and $6000 \mathrm{~cm}^{-1}$ (see the ESI $\uparrow$ ). The highest energy resonances, close to the ion-pair dissociation limit, are indicated by the extrapolated point close to the vertical axis $\left(E_{\mathrm{b}} \approx 25 \mathrm{~cm}^{-1}\right)$. Note that the point on the $y$-axis corresponds to the states shown in detail in Fig. 6 . Finally, the position of the crossings for $n=3$ and $n=4$ limits are indicated by arrows. 
interruptions by avoided crossings that predissociate parts of the progression. In the $\mathrm{H} \overline{\mathrm{H}}$ state, the potential has a potential maximum at $E_{\mathrm{b}} \sim 12000 \mathrm{~cm}^{-1}$ and the vibrational numbering to larger $E_{\mathrm{b}}$ is that of the outer well only, whose outer wall continues initially to have a dominantly coulombic potential.

\section{Conclusions}

The vibrational energy levels of the $\mathrm{H} \overline{\mathrm{H}}$ state of $\mathrm{H}_{2}$ have been calculated very close to dissociation, between the $n=3$ and $n=4$ asymptotes, under fully coupled conditions with the six other ${ }^{1} \Sigma_{\mathrm{g}}^{+}$states. All of the $\mathrm{H} \overline{\mathrm{H}}$ levels are subject to weak predissociation into the $\mathrm{H}+\mathrm{H}^{*}(n=3)$ channel and their widths indicate lifetimes $\sim 10$ ns $(n \sim 2000)$ arising from this. The absolute quantum defects associated with these levels are close to 64 and, with $n \sim 2000$, they clearly can be classified as heavy Rydberg states.

The calculations presented here complete the sequence of HR vibrational levels associated with the $\mathrm{EF}^{1} \Sigma_{\mathrm{g}}^{+}$and $\mathrm{HH}^{1} \Sigma_{\mathrm{g}}^{+}$states, the observed resonance structure and the higher $n^{1} \Sigma_{\mathrm{g}}^{+}$states that are bound by the Coulomb potential. This is the first report of HR states above the $n=4$ limit. HR behavior has now been observed from $\nu=0$ of the outer $\mathrm{F}^{1} \Sigma_{\mathrm{g}}^{+}$potential, through the vibrational levels of the $\mathrm{HH}^{1} \Sigma_{\mathrm{g}}^{+}$state and up to within $\approx 25 \mathrm{~cm}^{-1}$ of the ion-pair dissociation limit.

The analogous vibrational systems in the ${ }^{1} \Sigma_{\mathrm{u}}^{+}$manifold have been shown to have HR character from $\nu=0$ of the $\mathrm{B}^{1} \Sigma_{\mathrm{g}}^{+}$state, through the $\mathrm{BB}^{1} \Sigma_{\mathrm{u}}^{+}$state, and diabatically through the $n=3$ asymptote up to the limit of the published resonance structure. However, the region between the $n=4$ asymptote and the ionpair dissociation limit for the ${ }^{1} \Sigma_{\mathrm{u}}^{+}$manifold remains to be investigated.

Work is underway to include ionisation by coupling the inner-most region $(R<10$ a.u.) to the electronic continuum via a generalised $R$-matrix approach. The conceptual framework for incorporating ionisation and dissociation within an $R$-matrix formalism is already established. ${ }^{55-58}$ Judging by the present calculations, the effect of including ionisation on the line positions will be comparatively minor. In contrast, one should expect the effect on the line widths to be more significant, and a full account of the mixing with electronic Rydberg states will provide an understanding of how valence and electronic Rydberg states channel excitation intensity to heavy Rydberg states.

\section{Conflicts of interest}

There are no conflicts of interest to declare.

\section{Acknowledgements}

A. K. acknowledges support from a Royal Society of Edinburgh Sabbatical Fellowship (58507).

\section{References}

1 K. S. Kleinbach, F. Engel, T. Dieterle, R. Löw, T. Pfau and F. Meinert, Phys. Rev. Lett., 2018, 120, 193401. 
2 C. Asaro and A. Dalgarno, Chem. Phys. Lett., 1985, 118, 64-66.

3 S. Pan and F. H. Mies, J. Chem. Phys., 1988, 89, 3096.

4 E. Reinhold and W. Ubachs, Phys. Rev. Lett., 2002, 88, 013001.

5 R. C. Shiell, E. Reinhold, F. Magnus and W. Ubachs, Phys. Rev. Lett., 2005, 95, 213002.

6 E. Reinhold and W. Ubachs, Mol. Phys., 2005, 103, 1329-1352.

7 M. O. Vieitez, T. I. Ivanov, E. Reinhold, C. A. de Lange and W. Ubachs, Phys. Rev. Lett., 2008, 101, 163001.

8 M. O. Vieitez, T. I. Ivanov, E. Reinhold, C. A. de Lange and W. Ubachs, J. Phys. Chem. A, 2009, 113, 13237.

9 E. E. Marinero, R. Vasudev and R. N. Zare, J. Chem. Phys., 1983, 78, 692-699.

10 K. P. Lawley and R. J. Donovan, J. Chem. Soc., Faraday Trans., 1993, 89, 1885.

11 S. Yu and K. Dressler, J. Chem. Phys., 1994, 101, 7692.

12 L. Wolniewicz and K. Dressler, J. Chem. Phys., 1994, 100, 444.

13 E. Reinhold, W. Hogervorst and W. Ubachs, Phys. Rev. Lett., 1997, 78, 2543.

14 E. Reinhold, W. Hogervorst and W. Ubachs, Chem. Phys. Lett., 1998, 296, 411.

15 E. Reinhold, W. Hogervorst, W. Ubachs and L. Wolniewicz, Phys. Rev. A: At., Mol., Opt. Phys., 1999, 60, 1258-1270.

16 M. Cannon, C. Wang and F. Dunning, Chem. Phys. Lett., 2009, 479, 30.

17 S. Mollet and F. Merkt, Phys. Rev. A: At., Mol., Opt. Phys., 2010, 82, 032510.

18 M. Cannon, C. H. Wang, F. B. Dunning and C. O. Reinhold, J. Chem. Phys., 2010, 133, 064301.

19 A. Kirrander, J. Chem. Phys., 2010, 133, 121103.

20 A. Kirrander and Ch. Jungen, Phys. Rev. A: At., Mol., Opt. Phys., 2011, 84, 052512.

21 R. C. Ekey Jr and E. F. McCormack, Phys. Rev. A: At., Mol., Opt. Phys., 2011, 84, 020501.

22 R. J. Donovan, K. P. Lawley and T. Ridley, J. Chem. Phys., 2015, 142, 204306.

23 R. J. Donovan, K. P. Lawley and T. Ridley, Chem. Phys., 2015, 463, 145-148.

24 K. P. Lawley and R. J. Donovan, Chem. Phys. Lett., 2016, 659, 192-195.

25 A. M. Chartrand, R. J. Donovan, K. P. Lawley and E. F. McCormack, Chem. Phys. Lett., 2017, 676, 140-143.

26 Ch. Jungen, Handbook of High-resolution Spectroscopy, Wiley, 2010.

27 F. H. Mies, J. Chem. Phys., 1984, 80, 2514.

28 F. H. Mies and P. S. Julienne, J. Chem. Phys., 1984, 80, 2526.

29 T. S. Rose, M. J. Rosker and A. H. Zewail, J. Chem. Phys., 1988, 88, 6672-6673.

30 T. S. Rose, M. J. Rosker and A. H. Zewail, J. Chem. Phys., 1989, 91, 7415-7436.

31 S. E. Choi and J. C. Light, J. Chem. Phys., 1989, 90, 2593-2604.

32 V. Engel and H. Metiu, J. Chem. Phys., 1989, 90, 6116-6128.

33 S. Chapman and M. S. Child, J. Phys. Chem., 1991, 95, 578.

34 S. T. Cornett, H. R. Sadeghpour and M. J. Cavagnero, Phys. Rev. Lett., 1999, 82, 2488.

35 N. Balakrishnan, B. D. Esry, H. R. Sadeghpour, S. T. Cornett and M. J. Cavagnero, Phys. Rev. A: At., Mol., Opt. Phys., 1999, 60, 1407.

36 A. Kirrander, S. Rittenhouse, M. Ascoli, E. E. Eyler, P. L. Gould and H. R. Sadeghpour, Phys. Rev. A: At., Mol., Opt. Phys., 2013, 87, 031402.

37 M. Stenrup, Å. Larson and N. Elander, Phys. Rev. A: At., Mol., Opt. Phys., 2009, 79, 012713.

38 L. Wolniewicz, J. Chem. Phys., 1998, 108, 1499. 
39 T. F. Gallagher, Rydberg Atoms, Cambridge University Press, 1st edn, 1994.

40 F. Mrugala and D. Secrest, J. Chem. Phys., 1983, 79, 5960.

41 P. Quadrelli, K. Dressler and L. Wolniewicz, J. Chem. Phys., 1990, 92, 7461.

42 D. E. Manolopoulos, PhD thesis, University of Cambridge, Cambridge, 1988.

43 D. E. Manolopoulos, J. Chem. Phys., 1986, 85, 6425.

44 J. M. Hutson, Comput. Phys. Commun., 1994, 84, 1.

45 Ch. Jungen and F. Texier, J. Phys. B: At., Mol. Opt. Phys., 2000, 33, 2495.

46 C. H. Greene and Ch. Jungen, Adv. At. Mol. Phys., 1985, 21, 51.

47 T. Detmer, P. Schmelcher and L. S. Cederbaum, J. Chem. Phys., 1998, 109, 9694.

48 A. S. Dickinson and F. X. Gadea, Phys. Rev. A: At., Mol., Opt. Phys., 2002, 65, 052506.

49 A. S. Dickinson and F. X. Gadea, J. Mol. Struct.: THEOCHEM, 2003, 621, 87.

50 C. L. Pekeris, Phys. Rev., 1962, 126, 1470.

51 A. Dalgarno, Adv. Phys., 1962, 11, 281.

52 A. A. Radtzig and B. M. Smirnov, Reference Data on Atoms, Molecules, and Ions, Springer-Verlag, 1985.

53 G. Staszewska and L. Wolniewicz, J. Mol. Spectrosc., 2002, 212, 208.

54 L. Wolniewicz and K. Dressler, J. Chem. Phys., 1985, 82, 3292-3299.

55 A. Kirrander, H. H. Fielding and Ch. Jungen, J. Chem. Phys., 2007, 127, 164301.

56 A. Kirrander, H. H. Fielding and Ch. Jungen, J. Chem. Phys., 2010, 132, 024313.

57 A. Kirrander, Ch. Jungen and H. H. Fielding, Phys. Chem. Chem. Phys., 2010, 12, 8948.

58 A. Kirrander, Ch. Jungen and H. H. Fielding, J. Phys. B, 2008, 41(7), 074022. 